\title{
Ein Fall von chronischem Rotz beim Menschen.
}

Von Stabsarzt d. L. Dr. M. Heuer,

Chefarzt des Kriegsgefangenenlazaretts Hameln.

Dank der seuchenpolizeilichen Maßnahmen und ihrer strengen, gewissenhaften Durchführung gehören in Deutschland Rotzerkrankungen zu den Seltenheiten, und demgemäß sind auch der Veröffenthichungen in den letzten Jahrzehnten wenige; seit dem Jahre 1906 sind meines.Wissens nur zwei Arbeiten erschienen. Die eine von Wladi miroff(1), die andere von Martini und Besenbruch(2). Diese beschreibt eine chronische rotzartige Erkrankung beim Menschen aus dem Gouvernementslazarett von Tsingtau. Immerhin sind in den Jahren 1906-1912 in Deutschland zehn Todesfälle von Rotz vorgekommen, die sich nach Angabe der statistischen Mitteilungen des Kaiserhichen Gesundheitsamtes wie folgt verteilen: in den Jahren 1906, 1907, 1911 und 1912 je einer, im Jahre 1908 zwei und im Jahre 1909 vier Sterbefälle.

Im nachstehenden möchte ich einen im hiesigen Kriegsgefangenenlazarett zur Beobachtung und Sektion gekommenen Fall von chronischem Rotz beim Menschen mitteilen, der neben einigen Besonderheiten zeigt, ein wie vieldeutiges, die Diagnose erschwerendes Bild diese Krankheit zu bieten vermag. Der Hinweis hierauf erscheint mir gerade jetzt um so wichtiger, als bei der Möghichkeit, daß im Verlauf des Krieges noch weitere Einschleppung von Rotz stattfindet, die möghichst frühzeitige Erkennung der Seuche das erste Erfordernis ist zur Verhütung weiterer Ausbreitung. 
Die Krankengeschichte, einen Auszug aus dem Sektionsprotokoll sowie die Ergebnisse der bakteriologisch-kulturellen und pathologisch-anatomischen Untersuchungen stelle ich voran.

\section{Krankengeschichte.}

Leontin Ogarkoff, Gemeiner der 7. Kompagnie 19. Turkestanischen Infanterieregiments, Zivilberuf Landwirt aus Sarman, Kreis Samarskaja, geboren 3. Juli 1893, wird am 10. März 1915 in das Lazarett eingeliefert.

Befund am 11. März. Ziemlich kräftiger Mann, Temperatur 39, $4^{\circ}$, Puls 84, Atmung 20. Fïhlt sich seit mehreren Tagen krank mit Kopfschmerzen, Glieder- und Rückenschmerzen. Will vor etwa einem Jahr an Malaria in Turkestan gelitten haben. Befund: Zunge feucht, nicht belegt. Lunge hinten rechts bronchitische Geräusche. Urin frei von Eiweiß und Zucker. Kein Durchfall. Sonstige Organe nichts Abnormes. PriessnitzUmschlag. Aspirin.

22. März. Befund nicht geändert. Temperatur morgens $36,5^{\circ}$, abends 39, Puls 84, Atmung 20. Zunge etwas trocken, belegt. Kein Durchfall. Urin frei. Keine Roseolen. Milz nicht vergrößert. Wird zur Beobachtung auf Typhus in die Isolierbaracke verlegt.

24. März. Temperatur morgens 37 , abends $37,4^{\circ}$, Puls 72 , Atmungsorgane kein Krankheitsbefund. Zunge feucht, nicht belegt. Leib nicht aufgetrieben, Bauchdecken etwas straff. Verstopfung. Milz überragt etwas den Rippenbogen. Klagen über Schmerzen der ganzen linken Schädelseite, die beim Berühren sehr empfindlich ist, und in der unteren Kreuzbeingegend. Die Untersuchung des linken Ohres ergibt nichts Krankhaftes, die der Mundhöhle desgleichen. An den Beinen und im Gesicht rundliche, bis zweimarkstückgroße Narben, angeblich von Geschwüren herrührend (1914 Aufenthalt ein $J a h r$ in Turkestan, Malaria?). Am Nacken zahlrciche harte, kleine Drüsen fühlbar, desgleichen in beiden Leistenbeugen. Frühere Geschlechtskrankheiten verneint. Jodkalibehandlung.

29. März. Temperatur morgens $36,5^{\circ}$, abends $37,7^{\circ}$, Puls 72 . Die Kopfschmerzen auf der linken Seite sind verschwunden, angeblich noch Schmerzen in der Kreuzbeingegend und den Beinen. Aufsitzen im Bett und Gehen wird etwas zögernd ausgeführt. Pupillenreflex (Licht) ist vorhanden. Patellarreflex beiderseits lebhaft.

6. April. Temperatur morgens 37 , abends $37,6^{\circ}$, Puls 72 . Milzdämpfung reicht im Sitzen nach oben bis zum unteren Rand der siebenten Rippe, nach vorn $2 \mathrm{~cm}$ über den Rippenbogen hinaus, fühlbar. Sonst außer Schmerzen in der Kreuzbeingegend und den Beinen kein Krankheits befund.

13. April. Seit einigen Tagen heftige Schmerzen am oberen Teil des linken Wadenbeins, Schmerzen in der Kreuzbeingegend geringer, sonst derselbe Befund.

19. April. Temperatur $38,8^{\circ}$, Puls 104. Das ganze Gesicht ist hochgradig geschwollen, sodaß die Augen nur halb geöffnet werden können. Die Schwellung ist schmerzhaft, namentlich gegen Berührung, die Haut darüber gerötet. Die Grenzen der Schwellung verlieren sich allmählich. Urin klar, dunkelgelb, kein Eiweiß, kein Zucker. Milz überragt im Liegen dreifingerbreit den Rippenbogen. Schmerzen im linken Unterschenkel nur noch gering. Milzgrenzen im Sitzen oberer Rand siebente Rippe, hinten hintere Axillarlinie, vorn vordere Axillarlinie, unten $6 \mathrm{~cm}$ unterhalb des Rippenbogens. Chininbehandlung. (Erysipel?)

26. April. Temperatur morgens $38,5^{\circ}$, a bends $40,6^{\circ}$, Puls 108. Das Gesicht und der größte Teil des Kopfes sind geschwollen. Die Schwellung hat ihren Höhepunkt beiderseits über den Schläfen. Hier ist die Haut zum Teil blasenförmig abgehoben, ein Teil der Blasen ist gelbeitrig durchschimmernd, während die länger bestehenden Blasen zu braunem Schorf eingetrocknet sind. Die verschorften Partien sind zum Teil eingesunken und bilden eine geschwürig aussehende Fläche. Die geschwollenen Partien verlieren sich allmählich nach der gesunden Seite hin. Die Augen können wegen der Schwellung nur teilweise geöffnet werden. Auch auf der linken Schulter finden sich ähnliche schorfige und geschwürige Stellen wie an der rechten Schläfe. Die Blasen sind hier zum Teil dunkelblau (blutgefüllt). Die Umgebung ist stärker gerötet. Ueber den Lungen hinten unten Dämpfung, Knisterrasseln. Milztumor wie früher deutlich fühlbar. Mundschleimhaut blaß, an der linken Wange kleine, gelblich belegte Geschwürstelle. Zunge feucht, wenig belegt. Pneumonie, schwere Infektion.

28. April. Zunge trocken und belegt. Temperatur 38 , Puls 108. Der Befund im Gesicht und an der linken Schulter im wesentlichen unverändert. An beiden Beinen zahlreiche rote und blaurote, leicht geschwollene, umschriebene Stellen sowie einzelne bis erbsengroße Blasen, bläulich durchschimmernd. Leib mäßig aufgetrieben, etwas gespannt, nicht druckempfindlich. Durchfall.

30. April. Temperatur 39,80,37,5, Puls 96. Am Kopf und an der linken Schulter mit bräunlichem Schorf bedeckte Ulzerationen. Am linken Oberschenkel mehrfache blau durchschimmernde Blasen auf verödetem Grunde. Oedeme am Kopf, Skrotum und den Füßen. Bauchdecken straff gespannt, sodaß Bauchorgane nicht palpabel.
Ueber den Atmungsorganen rechts hinten unten Dämpfung und feuchtes, feinblasiges Rasseln, links hinten unten Schallverkürzung und Vesikuläratmen. Urin dunkelbraunrot, Spuren von Eiweiß, kein Zucker.

Es werden zur bakteriologischen Untersuchung an das Bakteriologische Institut Hannover (Geheimrat Frosch) eingesandt: 1. Inhalt von Bläschen am Oberschenkel, 2. Punktionsflüssigkeit aus den Oedemen des rechten Fußes, 3. Blutausstrichspräparat aus der rechten Ohrmuschel. Rotzverdacht.

3. Mai. Völlige Bewußtlosigkeit, Herzschwäche.

Tod erfolgt 3. Mai 1915.

Die Sektion ergibt außer den Befunden an der äußeren Leiche, wie sie bereits geschildert sind, folgendes Abnorme an inneren Organen Milz erheblich vergrößert, Kapsel zum großen Teil verdickt, mehr herdförmig als diffus. In beiden Lungen, namentlich rechts, zahlreiche scharf umgrenzte grünrote Herde von fester, an Verkäsung erinnernder $\mathrm{Be}$ schaffenheit. In der rechten Pleura mäßig viel Exsudat. Die Nasenschleimhaut (Rotzverdacht) weist keine sichtbaren Veränderungen auf.

Es werden Gewebsstücke der Lunge, der Milz und der Haut zur Untersuchung dem Pathologischen Institut Hannover (Professor Ströbe) übersandt.

Untersuchungsbefund des Bakteriologischen Instituts (Geheimrat Frosch): Im Bläscheninhalt wurden nur mikroskopisch, im Eiterinhalt (Hautgeschwür) mikroskopisch und kulturell rotzverdächtige Stäbchenbazillenge wonnen. Aus der Lunge, deren pneumonische Herde deutlich knötige Struktur zeigten, wurden ebenfalls mikroskopisch und kulturell rotzverdächtige Stäbchen gewonnen. Bei den Hautgeschwüren (Leiche) gelang das nicht wegen zahlreicher, offenbar sekundär hinzugetretener Eiterbakterien. Von den gewonnenen Kulturen aus den Hau tgeschwüren des noch lebenden Patienten und aus den pneumonischen Lungenstückchen der Leiche wurden im ganzen vier Meerschweinchen geimpft, in der für Rotz üblichenWeise, und zwei Kaninchen. Nach sechs Wochen starben die Meerschweinche nin schneller Aufeinanderfolge unter typischen, eindeutigen Erscheinungen des Rotzes. Bei den subkutan geimpften Kaninchen entstanden kraterförmige Geschwüre, die sich zurückbildeten. Später traten bei diesen Tieren geschwürige Arrosionen an der Nase auf, mit nässendem Auswurf. Auch die beiden Kaninchen sind gestorben. und wenig später unter gleichen Erscheinungen an der Nase zwei nicht geimpfte Kaninchen, die sich im gleichen Stalle befanden. Während der bakteriologische Nachweis von Rotzbazillen bei den verendeten Meerschweinchen ohne weiteres gelang; war dieses bei den Kaninchen nicht der Fall.

Die gewonnenen Kulturen waren in jeder Beziehung - also Form der Bazillen, Färbbarkeit(Gram-negativ), Wachstum auf Kartoffeln etc. für Rotz charakteristisch.

Pathologisches Institut (Prof. Ströbe):

Makroskopisch: Hautstück mit unregelmäßigen, tiefen, unterminierenden Geschwüren. Lungenstücke enthalten umschriebene, velblichrötliche, weiche, erbsen- bis pflaumengroße pneumonische Herde aon fast eitrigem Charakter. Milzstück enthält mehrere ziemlich feste, gusgesprochen keilförmige, anämische Infarkte, keine Abszesse.

Mikroskopisch: Hautgeschwüre zeigen stark eitrig infiltrierten Boden. Die Eiterung unterminiert die Nachbarhaut. Die eitrigen Partien neigen zur Nekrose. L unge: umschriebene Pneumonien. Das zellreiche eitrige Exsudat (polynukleäre Leukozyten) geht stellenweise in Nekrose über. Neben den Pneumonien sind auch kleine hämorrhagische Infarkte vorhanden. Milz: einfache anämische Nekrosen (Infarkte) nicht vereitert; daneben im Milzgewebe ganz seltene miliare Abszeßchen.

Es fandell sich zwar in der Lunge bei besonderer Färbung stellen. weise Gram.negative Bazillen, doch kann nicht behauptet werden, daß es Rotzbazillen waren. Dazu ist kultureller Nachweis erforderlich. .In den Lungenproben waren die gewöhnlichen Gram-positiven Eiterungen als Ursache dieser Herde nicht zu sehen.

Was zuerstdie A tiologie u nseres Falles betrifft, soläßt sich auch nur einigermaßen Sicheres nicht feststellen, wenn es auch wahrscheinlich ist, daß der Patient, der im Zivilberuf Landwirt war und als solcher mit Pferden umging, durch ein rotziges Pferd infiziert wurde. Ein mehrfaches Auftreten der Krankheit im Regiment konnte jedenfalls nicht nachgewiesen werden, und es ist daher nicht ausgeschlossen, $\mathrm{da} ß \mathrm{~B}$ er bereits infiziert eingezogen wurde. Ebensowenig finden sich für den Ort des Eintritts der Rotzbazillen in den Körper Anhaltspunkte. Die Anamnese ergab bei Aufnahme des Kranken in das Lazare keinerlei Verdachtsmomente auf Rotz. Er gab an, vor etwa einem Jahre in Turkestan an einer fieberhaften Erkrankung gelitten zu haben, die seiner Beschreibung nach für Malaria gehalten wurde. Erst später, als Geschwüre auftraten, konnte durch Befragen festgestellt werden, daß auch bei der ersten Erkrankung ähnliche Geschwüre bestanden, welche jene eigentümlichen, flächenförmigen, leicht eingezogenen, an Pocken erinnernden Narben zurückließen, die sich im Gesicht und an den Beinen fanden und über deren Ursprung bis dahin jeder Anhalt fehlte. Damit wurde die Mutmaßung einer überstandenen Malaria gegenstandslos. Ohne Zweifel hat 
es sich auch im Jahre 1914 um Rotz gehandelt, der damals in ein latentes Stadium überging, um jetzt von neuem aufzuflackern. Auf einen nicht ohne weiteres von der Hand zu weisenden Grund hierfür gedenke ich später zurückzukommen. - Der Verlauf der Krankheit selbst läßt sich in drei Abschnitte einteilen.

Der erste erstreckt sich über 14 Tage. Klagen allgemeiner Natur, leichte Bronchitis, gelegentliche Temperatursteigerungen, Fehlen jeglicher objektiv. nachweisbarer Organveränderungen kennzeichnen ihn, sodaß es fraglich erscheint, ob man überhaupt schon von einem Ausbruch der eigentlichen Rotzkrankheit reden darf.

Das zweite, die Zeit vom 22. März bis 19. April, also rund vier Wochen umfassende Stadium möchte ich als das einer Spontaninfektion bezeichnen, d. h. einer Zeit, wo die im Körper ruhenden Bazillen mobil werden und sich vermehren, ohne daß es zu einer lokalen Ansiedelung Irommt. Für diese Zeit ist bezeichnend die Milzschwellung und die für Rotz als Einleitung fast regelmäßig in der Literatur beschriebenen Schmerzen - auch auf Berühren - in den seitlichen Schädelpartien und in den Extremitäten mit Bevorzugung der unteren. Abgesehen von den ersten Tagen, finden wir keine Temperaturerhöhungen, und auch die subjektiven Erscheinungen klingen allmählich ab, sodaß der Patient zeitweise das Bett verlassen kann. Die anfangs gestellte Diagnose, Grippe, scheint sich zu bestätigen. Da plötzlich wird das Bild ein ganz anderes.

Die dritte Krankheitsepoche beginnt. Das Stadium der schweren Infektion mit allen Zeichen der Sepsis. Die durch die Blutbahn, wie Israel(3) nachwies, sich verbreitenden Bazillen haben den Körper überschwemmt und siedeln sich an verschiedenen Stellen der Haut und in der Lunge in Form von pneumonischen Herden an. Die Temperatur steigt zeitweise bis $40,0^{\circ}$, und nach 14 Tagen geht der Patient nach längerer völliger Bewußtlosigkeit unter den Erscheinungen der Herzschwäche zugrunde.

Wenden wir uns nach der Skizzierung des Gesamtbildes zu Finzelhciten des Falles, so ist besonders interessant die lange, vollständig symptomlose Zwischenzeit zwischen der ersten und zweiten Erkrankung. Wenn auch in der Literatur Fälle von weit längerer Dauer bekannt sind Bollinger(4) schildert einen 11 Jahre, Baracz(4) sogar einen 15 Jahre dauernden Rotzfall. - , so gehört doch ein derartiges etwa einjähriges, völliges Fehlen aller Krankheitserscheinungen, das sogar die Teilnahme an den Strapazen eines Feldzuges erlaubte, immerhin nicht zu den Alltäglichkeiten.

Ein ähnlicher Fall wird von M. Jakowski(5) beschrieben. Es handelte sich um einen Patienten, bei dem zwischen erstem und zweitem Auftreten der Krankheit drei Monate verstrichen, während deren er, wenn auch nicht schwer arbeiten, so doch außerhalb der Behandlung tätig sein konnte. Leider ließ sich der Endausgang der Krankheit nicht feststellen. Ich möchte daher auch nicht ohne weiteres die Krankheitserscheinungen der ersten Tage in unserem Falle als Rotzsymptome mit Sicherheit ansprechen, halte es, wie oben angedeutet, vielmehr nicht für ausgeschlossen, daß durch eine akute Grippe, welche den durch die Anstrengungen des Winterfeldzuges in seiner Widerstandsfähigkeit nicht unwesentlich herabgesetzten Körper anfiel, ein Frei- und Virulentwerden der ruhenden Bazillen begünstigt hat. Als sicheres erstes Symptom des Wiederauftretens der Rotzkrankheit trat die mit Temperatursteigerungen einhergehende Milzschwellung auf, die ich neben den bestehenden harten geschwollenen Lymphdrüsen und den aus der früheren fieberhaften Erkrankung resultierenden charakteristischen Narben bei chronischem Rotz für diagnostisch sehr wichtig halte. Ich möchte dieses betonen, trotzdem Strube(6) eine verhältnismäßig geringe Beteiligung der Lymphdrüsen für Rotz charakteristisch nennt mit der Angabe, daß auch Bollinger, Tardieu, Barány, Sommerbrodt und Katz auf das häufige Fehlen von Lymphdrüsenschwellungen aufmerksam machen. Vielmehr möchte ich, gestützt auf die von Strube(7) selbst, Kernig(8), Jakowski (5), Neisser(9), Zaudy (10), Martini und Besenbruch veröfentlichten Fälle, bei denen sich ebenfalls Lymphdrüsenschwellungen fanden, Niko laier (l. c.) beistimmen, der eine Beteiligung der Lymphdrüsen hervorhebt, allerdings unter besonderer Betonung der ,schmerzhaften, entzündlichen Schwellungen", die wohl mehr unter die Rubrik des akuten Krankheitsbildes einzureihen sind. Zwanglos ergeben sich aber hieraus die von uns beobachteten Drüsenknötchen, die ich nicht anstehe als Aufstapelungsort für die Rotzbazillen anzusprechen, um so mehr, als nach Nikolaier (l. c.) Rotzbazillen in Lymphdrüsen gefunden sind.

Die Fieber- und Pulskurve bot nichts Typisches. Die höchste erreichte Temperatur war $40,6^{\circ}$ mit 108 Pulsen, wie denn überhaupt die Pulsfrequenz von einer gegen die Schwere der sonstigen Erscheinungen geradezu abstechenden Geringfügigkeit war.

Ein die frühzeitige Diagnose und damit die erforderliche Isolierung erschwerender Umstand ist die auch in der Literatur fast überall auftauchende Vieldeutigkeit der ersten Erscheinungen bei Rotzerkrankungen. Auch in unserem Falle wurde genau so wie bei dem von $\mathrm{Zaudy}$ (l. c.) beschriebenen zuerst die Diagnose Grippe gestellt, dann, durch die wiederholten Temperatursteigerungen begründet, trat durch die Tatsache, daß mehrere Typhusfälle eingeliefert waren, begünstigt, Typhusverdacht auf und lie $B$ uns den Patienten auf die Isolierabteilung verlegen und damit für seine Umgebung unschädlich machen. Die nach Abklingen fast aller Erscheinungen auftretende Gesichtsschwellung ließ an Erysipel denken, wenn nicht die allmählich in die gesunde Umgebung ausstrahlende Röte der Schwellung dagegen gesprochen hätte.

Endlich, fast sieben Wochen nach Beginn der Erkrankung, führten die auftretenden Bläschen und Geschwüre auf den richtigen Weg, und es wurde eine bakteriologische Untersuchung eingeleitet. Von diesem Zeitpunkt ab nahm die Krankheit einen derartig rapiden Verlauf, daß sie jeder therapautischen Einwirkung spottete und auch die Bestätigung des Rotzverdachtes erst nach dem Tode des Patienten gelang. Späterhin sicherte dann das Ergebnis der angelegten Kulturen und der vorgenommenen Impfungen die eindeutige Diagnose: chronischer Rotz.

Literatur: 1. Rotz. Handb. f. pathog. Organismen 1913. - 2. Arch. f. Schiffs- u. Trop. Hyg. 1911. - 3. B. ki. W. 1883 . 4. Rotz. Handb. d. prakt Med. von Ebstein u. Schwaibe 1901, V. - 5. B. kl. W. 1891 - - 6. Arch, f. klin Chir. 1900. - 7. Charité Ann. 1894. - 8. Zschr. f. klin. Med. 1887. 9. B. k1. W. 1893 . - 10. D. m. W. 1900 . 